\title{
Birth of Pups after Intra-ovarian Bursal Transfer of Hamster Zygotes
}

\author{
Atsuo OGURA, Junichiro MATSUDA, Toshihiko ASANO and \\ Ryuzo YANAGIMACHI ${ }^{1)}$
}

\author{
Department of Veterinary Science, National Institute of Health, \\ 1-23-1 Toyama, Shinjuku-ku, Tokyo 162, Japan, and \\ ${ }^{1)}$ Department of Anatomy and Reproductive Biology, University of \\ Hawaii School of Medicine, Honolulu, Hawaii 96822, U.S.A.
}

\begin{abstract}
Syrian (Golden) hamster zygotes (pronuclear eggs) were collected from oviducts of donors and transferred to either oviducts or ovarian bursae of recipient females. Zygotes could develop into live young regardless of the post-ovulatory age of the zygotes and of the recipient females tested. The best results were obtained when the zygotes were transferred to ovarian bursae shortly before their first cleavage. Intra-ovarian bursal transfer of the zygotes is easy to perform. It may be applied to assessment of developmental potential of microsurgically operated hamster oocytes/zygotes.
\end{abstract}

Key words: Embryo transfer, Syrian hamster, Ovarian bursa, Oviduct, Zygote.

(J. Reprod. Dev. 41: 339-343, 1995)

$E^{n}$ mbryo transfer is a valuable research tool and an essential step in the production of offspring after in vitro manipulation of oocytes, zygotes, and/ or embryos. The steady technical improvement in the in vitro embryo culture and transfer has made it possible to produce chimeric, transgenic and cloned animals in several mammalian species.

The Syrian (golden) hamster has been used extensively in gamete biology because of the excellent features of their gametes suitable for the study of mammalian fertilization [1]. However, a very strong "two-cell block" we encounter during in vitro culture of hamster zygotes has prevented us from assessing the developmental capacity of in vitro manipulated oocytes/zygotes. Bavister and Arlotto [2] first succeeded in culturing hamster zygotes beyond the two-cell stage. Barnett and Bavister

Accepted for publication: September 22, 1995

Correspondence: A. Ogura
[3] obtained pups for the first time after in vitro fertilization and subsequent embryo transfer into recipient females. Despite steady improvement in the hamster zygote/embryo culture technique, the currently available technique is not yet dependable enough to evaluate developmental potential of microsurgically operated oocytes/zygotes.

As the native oviduct apparently provides the most favorable environment to developing embryos, the exposure of zygotes/embryos to culture media should be minimized until culture media and handling techniques are perfected. Hamster zygotes seem to be vulnerable to in vitro culture conditions. Sato and Yanagimachi [4] and Fukuda and Toyoda [5] were unable to obtain live young after oviductal transfer of hamster zygotes. According to Yang [6], hamster zygotes transferred into ovarian bursa were able to develop at least until the 13th day of pregnancy. Here, we compare the efficiency of hamster zygote transfei into 
the oviducts and ovarian bursae.

\section{Materials and Methods}

\section{Medium}

The medium used for zygote collection and transfer was the medium HECM-3 [3] as modified by Ogura and Yanagimachi [7]. Drops of the medium were covered with silicon oil in a plastic culture dish and equilibrated with $7.5 \% \mathrm{CO}_{2}$ in air at 37.5 C.

\section{Animals}

The Syrian (golden) hamsters purchased from National Cancer Institute (U. S. A.) and SLC (Japan) were used in this study. Ages of the females and males were 2 to 3 months and 4 to 8 months old, respectively. The animals were kept in an air-conditioned room with a $14 \mathrm{~h}$ light cycle (light, 0500 to $1900 \mathrm{~h}$ ). Females were examined every morning and the day of postovulatory vaginal discharge [8] was designated as Day 1 of the estrous cycle [9]. Under this light-dark condition females came into behavioral estrus in the evening of Day 4 and ovulated between 0100 and $0300 \mathrm{~h}$ of the next day (Day 1) [10]. When animals were mated fertilization in most eggs occurred between 0400 and 0600 $\mathrm{h}$ [10] and the first mitosis (cleavage) began after 1800 h of Day 1 (Fig. 1).

\section{Zygote collection}

Zygote donors were each injected with 20-30 I.U.
PMSG in the morning of Day 1 of the estrous cycle and mated with normal fertile males in the evening of Day 4 (Fig. 1). The next day (Day 1) donors were sacrificed at 1000, 1300 or $1600 \mathrm{~h}$. Excised oviducts were flushed with a small quantity of warm (30-37 C) HECM-3. For zygote collection at $1000 \mathrm{~h}$, the flushing medium contained $2 \mathrm{mg} / \mathrm{ml}$ hyaluronidase (300 USP units/mg, ICN Biochemicals, Costa Mesa, CA) and $3 \mathrm{mg} / \mathrm{ml}$ BSA (fraction V, Miles Diagnostics, Kankakee, IL). Hyaluronidase was used to disperse cumulus cells from zygotes. This enzyme was not necessary for flushing zygotes at 1300 and $1600 \mathrm{~h}$ because cumulus cells had already been dispersed.

\section{Recipient females}

A group of recipients was mated with vasectomized males in the evening of Day 4 of the estrous cycle. They received zygotes from donors on the following day (Day 1). Another group of recipients received zygotes on Day 4 of the estrous cycle, then mated with vasectomized males in the evening of the same day. The former was called "synchronous zygote transfer", the latter "asynchronous zygote transfer". In some experiments, zygotes from albino parents were transferred into foster mothers of wild type (brown coat and black eyes) for easy identification of the genetic origin of the pups.

\section{Zygote transfer}

Either Day 1 (synchronous) or Day 4 (asynchronous) of the estrous cycle recipient females were

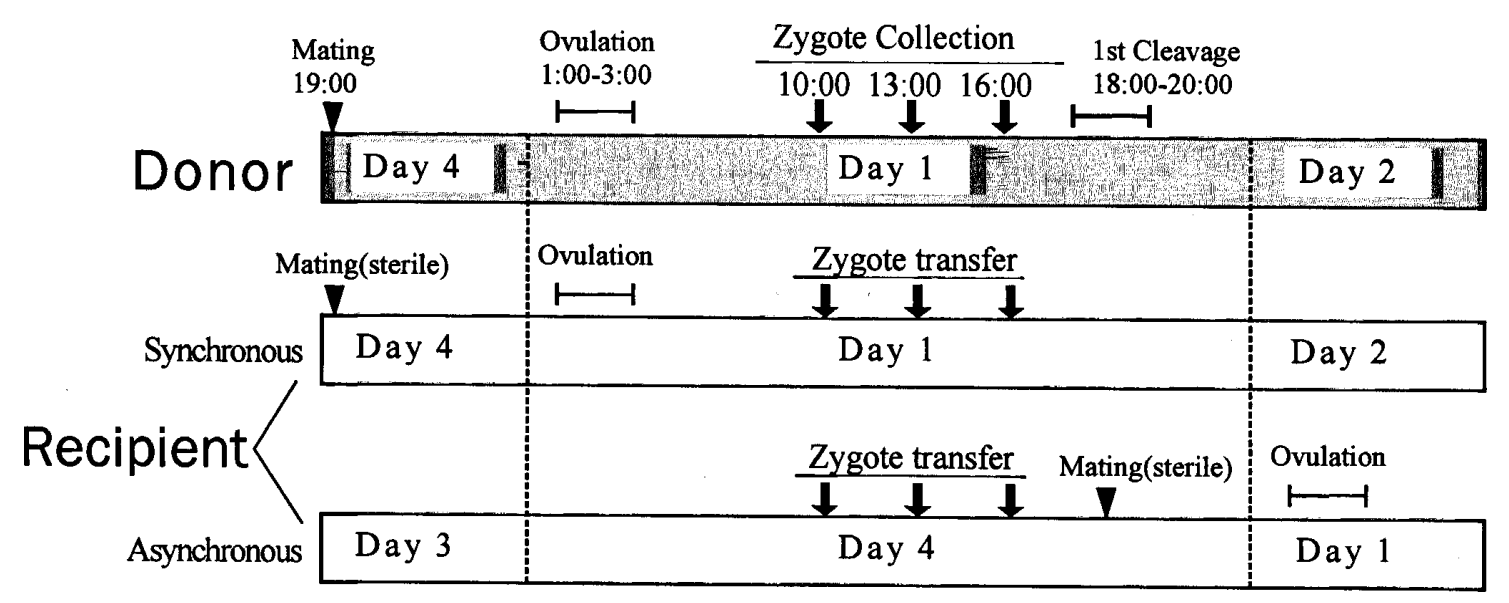

Fig. 1. Diagram showing the schedule of zygote collection and transfer in synchronous and asynchronous zygote transfer. 
anesthetized by an intraperitoneal injection of sodium pentobarbital $15 \mathrm{~min}$ prior to zygote transfer. The ovary and oviduct of one side (either right or left) were first exposed through a dorso-lateral incision, about $1.5 \mathrm{~cm}$ in length. Eight to 12 zygotes were transferred into the ovarian bursa or oviduct using a glass pipette (120-140 $\mu \mathrm{m}$ in inner diameter), together with a small amount of the medium (120-150 nl). In a series of experiments, the zygotes were transferred into the ovarian bursa. Tips of bursal transfer pipettes were broken with a fine forceps so that the sharp edge easily pierced the wall of the bursa. The pipette pierced through a fat-pad around the ovary, then through the ovarian bursa until the tip of the pipette was clearly visible through the thin, transparent ovarian bursal wall. In another experiment series, a small incision was made on the wall of the ovarian bursa and the tip of the pipette was inserted through the infundibulum into the ampullary region of the oviduct, where the zygotes were released. Transfer of zygotes into both sides of the ovarian bursae or oviducts was completed within $30 \mathrm{~min}$ after collection of zygotes from donor females. Recipient females were examined for postestrus vaginal discharge on Day 5, and those with discharge were considered "failed to have activated corpa lutea" and were eliminated from the data. Recipients that failed to become pregnant resumed estrous cycle and showed the vaginal discharge on Day 9 or 10 [8]. The remaining animals were allowed to deliver young. After weaning of pups, the mothers were sacrificed and the implantation sites (placental scars [11]) were counted to estimate the total number of embryos implanted.

In a separate series of experiments, the oviducts of unmated animals were flushed with the medium $24 \mathrm{~h}$ after zygote transfer into the ovarian bursa to determine the efficiency of zygote transport into the oviduct. Embryos (one- to four-cells) with sperm tails within the ooplasm were considered to be those transferred into the ovarian bursa.

Data were analyzed using the $\chi^{2}$-test.

\section{Results}

The results of zygote transfer into the oviducts and ovarian bursae are summarized in Tables 1 and 2. Although pups were born in all groups, the best results were obtained when zygotes were transferred into the ovarian bursae of recipients shortly before their first cleavage $(1600 \mathrm{~h})$. Bursal transfer of zygotes were superior to oviductal transfer for the production of live pups (Table 1). The postovulatory age of zygotes appears to affect the

Table 1. Birth of hamster pups after transfer of zygotes into the oviducts or ovarian bursae

\begin{tabular}{llccrr}
\hline & & & \multicolumn{2}{c}{ Production rate of pups } & \\
Site of transfer & Type of transfer & Pregnancy rate & Pregnant recipients & Overall & Implantation rate \\
\hline Oviduct & Synchro. & $2 / 5(40)^{\mathrm{a}}$ & $10 / 40(25)$ & $10 / 100(10)^{\mathrm{a}}$ & $15 / 100(15)^{\mathrm{a}}$ \\
Oviduct & Asynchro. & $2 / 5(40)^{\mathrm{a}}$ & $6 / 40(15)^{\mathrm{a}}$ & $6 / 100(6)^{\mathrm{a}}$ & $6 / 100(6)^{\mathrm{a}}$ \\
Bursa & Synchro. & $5 / 5(100)^{\mathrm{b}}$ & $27 / 99(27)$ & $27 / 99(27)^{\mathrm{b}}$ & $38 / 99(38)^{\mathrm{b}}$ \\
Bursa & Asynchro. & $5 / 5(100)^{\mathrm{b}}$ & $32 / 88(36)^{\mathrm{b}}$ & $32 / 88(36)^{\mathrm{b}}$ & $41 / 88(47)^{\mathrm{b}}$ \\
\hline
\end{tabular}

Zygotes were collected from the oviducts at $1600 \mathrm{~h}$ and transferred within $30 \mathrm{~min}$.

a, bDifferences within columns $(\mathrm{P}<0.05)$.

Table 2. Birth of hamster pups after intrabursal transfer of zygotes of different ages

\begin{tabular}{ccccc}
\hline & & \multicolumn{2}{c}{ Production rate of pups } & \\
\cline { 3 - 4 } Time of transfer & Pregnancy rate & Pregnant recipients & Overall & Implantation rate \\
\hline $10: 00$ & $4 / 5(80)$ & $21 / 84(25)^{\mathrm{a}}$ & $21 / 104(20)^{\mathrm{a}}$ & $26 / 104(25)^{\mathrm{a}}$ \\
$13: 00$ & $4 / 5(80)$ & $16 / 91(18)^{\mathrm{b}}$ & $16 / 113(13)^{\mathrm{a}}$ & $25 / 113(17)^{\mathrm{a}}$ \\
$16: 00^{*}$ & $5 / 5(100)$ & $32 / 88(36)^{\mathrm{a}}$ & $32 / 88(36)^{\mathrm{b}}$ & $41 / 88(47)^{\mathrm{b}}$ \\
\hline
\end{tabular}

*Same as asynchronous intrabursal transfer in Table 1.

a, $b$ Differences within columns $(\mathrm{P}<0.05)$. 
production rate of offspring after intrabursal transfer (Table 2).

Twenty-four hours after intrabursal transfer, $62 \%$ $(48 / 78)$ and $57 \%$ (54/94) of the transferred embryos were recovered from synchronous and asynchronous recipients (four females each), respectively. The difference between synchronous and asynchronous transfer was not significant $(\mathrm{P}>0.05)$.

\section{Discussion}

Under the lighting conditions we used, hamsters ovulated between 0100 and $0300 \mathrm{~h}$ of Day 1 of the estrous cycle [10]. Therefore the zygotes collected from donors and transferred to recipients were 7 to $15 \mathrm{~h}$ after ovulation. In synchronous transfer, the post-ovulatory ages of the donor's zygotes and the recipient's oviducts were the same, whereas in asynchronous transfer, donor's zygotes were one day older than the recipient's oviducts. In embryo transfer in the mouse $[12,13]$ and hamster [5], the highest pregnancy rate was obtained when donor's embryos were one day older than recipient's oviducts or uteri. Apparently, this was not the case in the hamster zygote transfer we performed in the present study. The rates of the embryos that developed to term were not statistically different between synchronous and asynchronous zygote transfer groups (see Table 1). Also we found no significant difference between synchronous and asynchronous recipients in the rate of embryos transported into the oviducts within $24 \mathrm{~h}$ after intrabursal transfer.

It should be noted that the zygotes collected from the oviducts at $1600 \mathrm{~h}$ of Day 1 developed to postimplantation stages or to term better than those collected at 1000 and $1300 \mathrm{~h}$ of Day 1. Although the zygotes at $1000 \mathrm{~h}$ and $1600 \mathrm{~h}$ of Day 1 looked alike in their appearance, those at $1600 \mathrm{~h}$ seemed to withstand in vitro handling procedures better than younger zygotes. Barnett and Bavister [3], who cultured hamster zygotes in vitro, noticed that the zygotes collected from the oviducts shortly before the first cleavage could develop better in vitro than those collected long before the expected time of the first cleavage.

Hamster zygotes seem to be very sensitive to environmental changes. Sato and Yanagimachi [4], who used medium TC199 for embryo transfer, could not obtain full term embryo after zygote transfer into oviducts. Perhaps medium TC199 they used was detrimental to the hamster zygotes because it contains phosphate and glucose [14]. We obtained live pups after zygote transfer by using HECM-3, although the medium seems to be still suboptimal for hamster zygotes, particularly for those soon after fertilization. Further improvement of zygote-handling media is definitely needed.

Successful fertilization and embryonic development can occur following transfer of unfertilized oocytes into ovarian bursae of the rat [15] and hamster [16]. The presence of cumulus cells around the oocytes is not absolutely essential ([15] and the present study), but seems to assist oocyte pickup by the fimbria [17].

The reason why the ovarian bursal transfer of cumulus-free hamster zygotes was superior to the oviductal transfer is not clear at present. The infundibulum and / or the upper region of the oviduct could have been damaged by insertion of a transfer pipette into the oviduct. Technically speaking, zygote transplanted into the ovarian bursa is much simpler and easier than the oviductal transfer. In this respect, Yang [6] should be credited for the first application of this technique to embryo (zygote) transfer. It would be interesting to know whether this technique can be applied to other animals with ovarian bursae (e.g., mouse and rats).

We have succeeded in producing live young after transferring in vitro fertilized hamster oocytes into ovarian bursae of recipient females, although the success rate is rather low at present (Ogura and Yanagimachi, unpublished data). Sperm injected hamster oocytes developed into three- to four-cell embryos after intrabursal transfer (Ogura and Yanagimachi, unpublished data).

\section{References}

1. Cherr GN, Drobnis EZ. Fertilization in the golden hamster. In: Dunbar, BS, O'Rand MG (eds.), Comparative Overview of Mammalian Fertilization,
New York: Plenum Press; 1991: 217-243.

2. Bavister BD, Arlotto T. Influence of single amino acids on the development of hamster one-cell em- 
bryos in vitro. Mol Reprod Dev 1990; 25: 45-51.

3. Barnett DK, Bavister BD. Hypotaurine requirement for in vitro development of golden hamster one-cell embryos into morulae and blastocysts, and production of term offspring from in vitro-fertilized ova. Biol Reprod 1992; 47: 297-304.

4. Sato A, Yanagimachi R. Transplantation of preimplantation hamster embryos. J Rerpod Fertil 1972; 30: 329-332.

5. Fukuda Y, Toyoda Y. Development of normal young after asynchronous transfer of 2-cell hamster embryos. Jpn J Zootech 1983; 54: 805-811.

6. Yang WH. Induction of lordosis, ovulation and pregnancy by a single injection of hCG in the hamster. Endocrinol 1971; 89: 287-293.

7. Ogura A, Yanagimachi R. Round spermatid nuclei injected into hamster oocytes form pronuclei and participate in syngamy. Biol Reprod 1993; 48: 219-225.

8. Orsini MW. The external vaginal phenomena characterizing the stages of the estrous cycle, pregnancy, pseudopregnancy, lactation, and the anestrous hamster, Mesocricetus auratus Waterhouse. Proc Anim Care Panel 1961; 11: 193206.

9. Greenwald GS. Analysis of superovulation in the adult hamster. Endocrinol 1962; 71: 378-389.
10. Smith TT, Koyanagi F, Yanagimachi R. Distribution and number of spermatozoa in the oviduct of the golden hamster after natural mating and artificial insemination. Biol Reprod 1987; 37: 225-234.

11. Orsini MW. The vascular knot of the hamster uterus. J Morphol 1957; 100: 565-599.

12. McLaren A, Michie D. Studies on the transfer of fertilized mouse eggs to uterine foster-mothers. I. Factors affecting the implantation and survival of native and transferred eggs. J Exp Biol 1956; 33: 394-416.

13. Wiebold JL, Anderson GB. The effect of recipient age on the success of embryo transfer in mice. Lab Anim Sci 1986; 36: 161-163.

14. Schini SA, Bavister BD. Two-cell block to development of cultured hamster embryos is caused by phosphate and glucose. Biol Reprod 1988; 39: 11831192.

15. Walton EA, Armstrong DT. Oocyte normality after superovulation in immature rats. J Reprod Fertil 1983; 67: 309-314.

16. Yanagimachi R. Developmental ability of precociously superovulated golden hamster eggs. Gamete Res 1984; 9: 231-237.

17. Mahi-Brown CA, Yanagimachi R. Parameters influencing ovum pickup by oviductal fimbria in the golden hamster. Gamete Res 1983; 8: 1-10. 